\title{
Fleiner, Micha (2016): Performancekünste im Hochschulstudium. Transversale Sprach-, Literatur- und Kulturerfahrungen in der fremdsprachlichen Lehrerbildung.
}

\author{
Berlin: Schibri. 335 Seiten. ISBN 978-3-86863-171-5
}

\section{Sandrine Eschenauer}

\author{
Der Mensch kommt nicht dahin, \\ sich durch seine intellektuelle Bildung wirklich zu veredeln, \\ wenn diese nicht auf die vollendete Ausbildung seines Herzens gebaut ist. \\ (Pestalozzi, Sämtliche Werke 14/349)
}

Es ist zwischen Wissen und Tun ein himmelweiter Unterschied.

Wer aus dem Wissen allein sein Handwerk macht, der hat wahrlich groß Acht zu geben, dass er das Tun nicht verlerne.

(Pestalozzi, Sämtliche Werke 2/125)

Diese Doktorarbeit positioniert sich als evidenzbasierte Forschung („evidencedbased contribution"), die die Entwicklung und Förderung performativer Ansätze in der (Fremdsprachen)lehrerausbildung begründet und rechtfertigt.

\section{Präambel ...}

Das Theater wurde als Unterrichtsmedium schon vor vielen Jahrhunderten anerkannt und verwendet, insbesondere in der Antike von Aristoteles. Auch später spielten ästhetische Ansätze im Bereich der Pädagogik immer eine wichtige Rolle, wie zum Beispiel im Kontext des Pragmatismus (James, Dewey) und der Reformpädagogik (Reichwein, Steiner, Montessori etc.). Weit entfernt von einer reinen ästhetischen Lehre oder einem Schönheitskanon, die seit jeher umstritten waren und heftig diskutiert wurden, ist die Ästhetik im Rahmen einer aktivierenden Pädagogik ,in den Grundelementen“ zu suchen, „bei den Ereignissen und Szenen, die das aufmerksame Auge und Ohr des Menschen auf sich lenken, sein Interesse wecken und, während er schaut und hört, sein Gefallen aufrufen" (Dewey 1934/2014: 11). In Deutschland wurden diese Erziehungsansätze, die die Erfahrung als Grundlage der Erkenntnis eingebracht haben, im Theaterbereich insbesondere formalisiert durch die Einrichtung einer Dozentenstelle für das Schulspiel an der Pädagogischen 


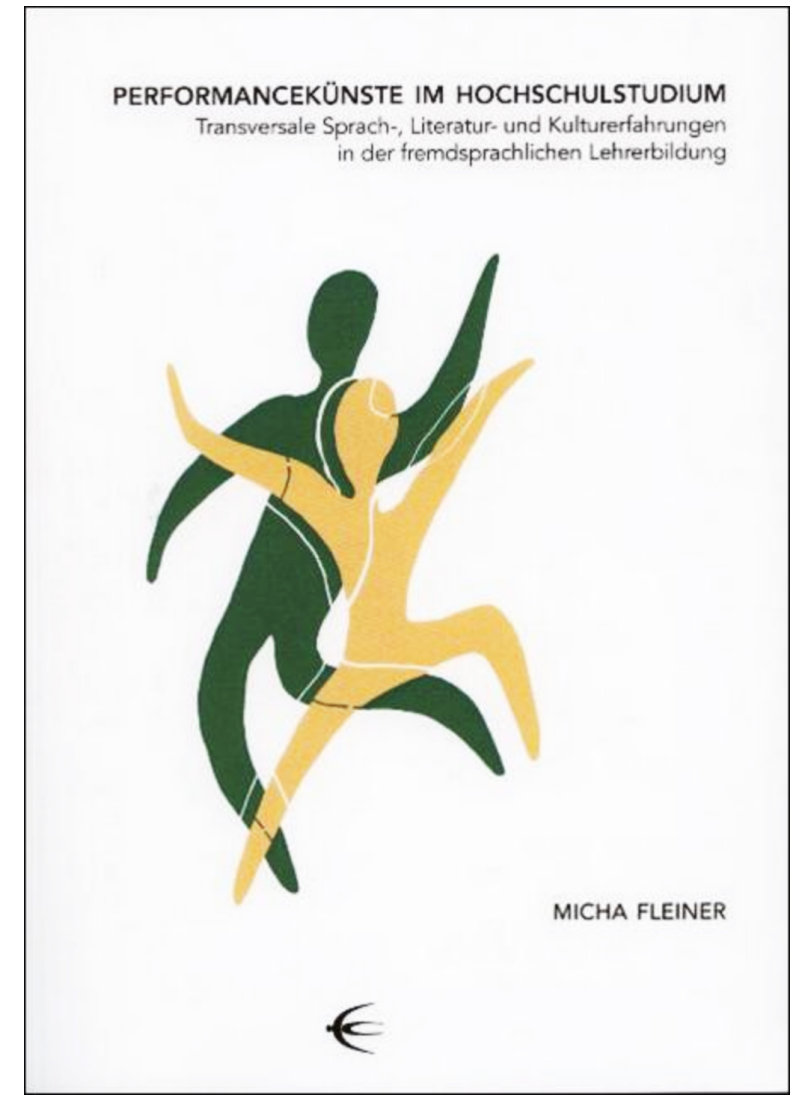

Abbildung 1: //www.ucc.ie/en/scenario/scenariobooks/

Hochschule Berlin im Jahre 1963. Ihr Begründer, Hans-Wolfgang Nickel, bot damals eine Ausbildung an, die aus dem Schauspiel als Handlungserfahrung des Schauspielers schöpfte. Inhaltliche Komponenten waren zum Beispiel die Sprecherziehung, das Darstellende Spiel - und darüber hinaus auch Fächer, die das Spiel als Grundstein des Lernprozesses verstanden, von der Schauspielkunst bis hin zu Lerninhalten und zur sozialen Kompetenz. Dieser Lehrstuhl wurde in den 70er Jahren, insbesondere unter Leitung der Professoren Nickel und Ritter, zur Theaterpädagogik weiterentwickelt. Sie wurde offiziell mit dem Fremdsprachenunterricht verknüpft, als die erste Doktorarbeit zum Einsatz von Drama-Techniken im fremdsprachlichen Lehren und Lernen veröffentlicht wurde (Schewe 1993), in der vor allem auch eine Auseindersetzung mit der Dramapädagogik, dem britischen Äquivalent zur deutschen Theaterpädagogik, stattfand.

\section{Die Publikation...}

Zwanzig Jahre später verschiebt Micha Fleiner den Fokus und nimmt einen analytischen Abstand gegenüber dem Einsatz performativer Künste in der Französischlehrerausbildung. In seiner 2016 veröffentlichten Doktorarbeit 
Performancekünste im Hochschulstudium. Transversale Sprach-, Literatur- und Kulturerfahrungen in der fremdsprachlichen Lehrerbildung stellt er die Ergebnisse seiner sowohl quantitativen als auch qualitativen Analyse im Fach- und Studienbereich Romanistik für die Lehrerausbildung im deutschsprachigen Bereich (Deutschland, Österreich, Schweiz) vor. Dabei greift er Elemente der Dramapädagogik auf. Er hinterfragt ihre Auswirkung auf Universitätsebene, indem er Dozentenbefragungen analysiert; und im Bereich der Schule, indem er Interviews mit Studenten durchführt. Diese angehenden Lehrer kommentieren sowohl ihre Erfahrungen mit performativen Ansätzen im Bereich der Lehrerausbildung als auch deren (konkrete oder erhoffte) schulpraktische Anwendung in ihrem eigenen Unterricht. Fleiner sieht den Hauptunterschied zwischen traditionellem und dramaorientiertem Fremdsprachunterricht in der ästhetischen und ganzheitlichen Orientierung des letzteren,

(...) in dem die dramatische Kunst (insbesondere als Theaterkunst, aber durchaus im Zusammenspiel zwischen andere Kunstformen wie zum Beispiel Film, Performance Art, Storytelling, Oper) zur Inspirationsquelle und zur Orientierung für das pädagogische Handeln wird. Zugrunde liegt ein Menschenbild, welches den Lernenden als ganzen Menschen erst nimmt. (...) Das im Adjektiv ,dramapädagogisch' enthaltene Wort ,drama' geht etymologisch auf griechisch dran in der Bedeutung ,tun, handeln' zurück und hebt damit den Aspekt der (...) Handlungsorientierung besonders hervor. (Schewe 2010, zitiert in Fleiner 2016: 48)

Diese Publikation bietet einen wertvollen Einblick in die Möglichkeiten - ja sogar in die Notwendigkeit - performative Lehr-Lernansätze in den universitären und schulischen Kontext systematischer zu implementieren, und zwar insbesondere in der Fremdsprachenlehrerausbildung. Deren Auswirkungen werden aus drei Perspektiven betrachtet: Methodologie, Lernen und Forschung. Durch das Zusammenspiel von performativen und pädagogischen Wissenschaften entstehen neue Fragestellungen. Der erfahrungsorientierte und ästhetische Charakter der performativen Künste übersteigt einen rein intellektuellen Ansatz, der die leiblich/körperliche Dimension der Erkenntnis vernachlässigt. In dieser von Fleiner privilegierten fachlich-methodischen Annäherung zwischen Dramapädagogik und Fremdsprachenlehre soll im Gegenteil eine Praxis entstehen, die die sinngebenden Modalitäten im Lehr- und Lernbereich von Sprachen mal aus einer praktischen, mal aus einer wissenschaftlichen Perspektive bestimmt.

Das Buch besteht aus neun Kapiteln. Im ersten Teil formuliert der Autor seine Problematik. Inwiefern könnten kreative, auf Performance-Künste basierende Ansätze die Hochschulausbildung bereichern?

Der Autor merkt an, dass in den letzten Jahrzehnten, insbesondere im englischsprachigen Bereich, zahlreiche Publikationen auf den Markt gebracht wurden, die die positiven Auswirkungen von künstlerischer Praxis im Fremdsprachenunterricht darstellen. Er zitiert insbesondere Manfred Schewe, der sich auf Dramapädagogik in der Sprach-, Literatur- und Kulturvermittlung spezialisiert hat und als Vorreiter dieser ästhetisch-performativen Ansätze im 
Kontext der Fremdsprachenbildung in Deutschland gilt. Andere Standpunkte, die auf verschiedenen theoretischen Grundlagen basieren und entweder als Inspirationsquelle galten oder parallel entstanden, werden außerdem von Fleiner aufgelistet. Zu erwähnen sind hier die Vorschläge, dramapädagogische Ansätze im Rahmen der neuesten neurowissenschaftlichen Erkenntnisse (Sambanis) in verschiedenen Bereichen einzusetzen, etwa bei der Vermittlung von Grammatik und zur Förderung von sprachlicher Korrektheit (Even), zur Förderung aisthetisch-sinnlichen (Huber) und interkulturellen Lernens (Kessler) und speziell auch in der Lehrerausbildung (Lutzker). Im Feld der Romanistik hebt Fleiner besonders die Bedeutung von Dufeus und Feldhendlers Werk hervor. Weitere zahlreiche Veröffentlichungen, die diese Promotionsarbeit beeinflusst haben, finden hier auch Erwähnung, insbesondere aus der Theaterpädagogik (Koch) und der Dramapädagogik (Schewe) stammend.

Fleiner schöpft aus diesen Studien und deren Lehre für seine Perspektive auf die mögliche Entwicklung der Aus- und Fortbildung und der fremdsprachendidaktischen Forschung.

Im zweiten Kapitel widmet sich der Autor dem theoretischen Rahmen dieser Problematik und definiert vier Schlüsselbegriffe: Ästhetik (33), Performativität (37), transversale Schnittstellen (41) und Dramapädagogik (47).

Ästhetik: Fleiner geht es hauptsächlich darum, die lebenden Sprachen zunächst als Kommunikationsmittel zu verstehen, die in der Interaktion Welten öffnen und damit unterschiedliche Sichtweisen und Interpretationen der realen Gegebenheiten ermöglichen. Die künstlerische Praxis entfaltet diese multiple Wahrnehmung und das damit verbundene divergente, transformatorische Denken. Die Theater- und/oder Dramapraxis, indem sie intrinsisch verschiedene Kunstformen integriert, erweist sich als besonders effizient. Durch eine ästhetisch orientierte Spracherfahrung sprengt der Unterricht die Grenzen der normierten Schemata von Frage-Antwort-Katalogen, Textinterpretationen, Meinungsäußerung oder didaktisierten Dialogen, die gewöhnlich im fremdsprachlichen Kontext als Grundmodell für die Kommunikationskompetenz gelten. Nach Fleiners Meinung wäre es im Gegensatz dazu wünschenswert, eine „polyästhetische“ und „dynamische“ (35) Entwicklung in den Bildungswissenschaften zu fördern, die eigentlich der Kommunikation innewohnt, sofern Ästhetik als Lebenserfahrung verstanden wird - d.h. als ein komplexer Zusammenhang von Phänomenen. Es erweist sich somit nach Fleiner als unmöglich, eine einzige Definition von Ästhetik anzubieten. Vielmehr sollten die verschiedenen Wahrnehmungen und Begrifflichkeiten der Ästhetik in ihrem jeweiligen Verwendungskontext betrachtet werden, um nicht ,gegen die Phänomene zu verstoßen" (Welsch (1996), zitiert in Fleiner 2016: 34). In diesem Kontext geht es eher darum, die ästhetische Dimension des Unterrichtens und Lernens und mit ihr „Phänomene, Prozesse und Produkte in ihrer umfassenden Wirkung und Inszeniertheit" $\mathrm{zu}$ verstehen und zu fördern (Sting (2003), zitiert in Fleiner 2016: 35). Durch eine systematischere Anwendung performativer Lehr- und Lernformen strebt Fleiner eine temporäre „Wiederverzauberung der Welt" an (Fischer-Lichte (2004), zitiert in Fleiner 2016: 35); eine Verzauberung im 
Unterricht, die „sich in der Verknüpfung von Kunst und Leben vollzieht“ (ibid.); eine Verzauberung, die in der Lage sei, „das Affektive, die Emotionalität und das Imaginative aufzunehmen" (Böhme (2013), zitiert in Fleiner 2016: 35); „eine Verzauberung schließlich, die ,die Sehnsucht nach einer anderen Präsenz' (Welsch 2012: 12) zu stillen imstande ist, nämlich die Sehnsucht ,nach der unwiederholbaren Präsenz hic et nunc, nach dem singulären Ereignis'“ (Fleiner 2016: 35). Fleiners Verständnis von Ästhetik als theoretischer Rahmen für den Fremdsprachenunterricht wird vor allem von Dewey und Welsch geprägt. Es basiert auf der Beziehung zu erlebten Momenten, die einen emotionalen Einsatz offenbart und Aufmerksamkeitsmechanismen in Gang setzt. Ästhetische Erfahrung, so Fleiner, könne die Aufmerksamkeit der Lernenden hervorrufen und dabei fruchtbare Lernprozesse entfalten, die sowohl mit Emotionen als auch mit mentaler Reflexion verbunden sind.

Performativität: Fleiner beruft sich auf Austins (1962/1972) Schriften, für den die Hauptfunktion der Sprache darin besteht, auf die Welt einzuwirken - und nicht darin, sie zu beschreiben. Fleiner zitiert ebenso Fischer-Lichte $(2005$; 2012), die das Augenmerk besonders auf die körperliche Dimension der Sprache als sinnstiftende Ausdrucksform und Handlungsdarstellung richtet. Fleiner betont folglich den eminent performativen Charakter der verbalen und nonverbalen Kommunikationsprozesse. Die Performanz-Leistung der Lernenden sollte nach ihm an ihrer Fähigkeit gemessen werden, Sinn im gemeinsamen Handeln entstehen $\mathrm{zu}$ lassen, statt $\mathrm{zu}$ evaluieren, ob sie Situationen, Gegenstände, Texte etc. nennen und beschreiben können. Für die von Fleiner zitierten Autoren ist die Performanz ein einverleibtes Medium des Wissens - eine Welterkenntnis, die in unseren Körpern ist und durch sie Ausdruck findet. Es handelt sich dabei um das Wesen menschlicher Kommunikation.

Die Praxis des Dramas ermöglicht eine performative - gemeint ist damit eine einverleibte (embodied), sensorische, soziale und intellektuelle (Wulf \& Zirfas 2007, zitiert in Fleiner 39) -, Fragen stellende Lernform im fremdsprachlichen Unterricht und ermöglicht dabei auch effiziente Zugänge zur Syntax, Lexik, Prosodie, Phonetik etc.).

Transversale Schnittstellen: Die erwähnte körperliche Dimension des Lernens und des Wissens, so bemängelt es Schewe (2011), findet nur selten oder gar keinen Platz im Hochschulbereich. Somit wird Fleiners Vorschlag besonders relevant, „crosscurriculare“ Bildungsmodule zu implementieren, die Fremdsprachen und Künste verbinden und performativ ästhetische Elemente beinhalten, die wiederum eine vermittelnde Rolle zwischen den Fächern spielen könnten.

Dramapädagogik: Theater- und Dramapädagogik sind nur schwer definierbar und voneinander unterscheidbar, da die entsprechenden historischen Bewegungen dieser Studienfächer und Praxisfelder auf internationaler Ebene sehr unterschiedlich umgesetzt werden (théâtre en éducation, drama in education, etc.) (Streisand (2012), zitiert in Fleiner 2016: 49). Fleiner entwickelt sein Verständnis des Begriffs im Laufe der Kapitel.

Das dritte Kapitel liefert sodann einen quantitativen Überblick über die 
dramapädagogische Praxis im Hochschulbereich in den Ausbildungsgängen des Master of education für das Lehramt für Französisch in Deutschland, in Österreich und in der Schweiz. Lediglich eine geringe Zahl der Angebote weisen dramapädagogische Elemente in der Ausbildung auf (13,66\% der 61 befragten Universitäten).

Die Kapitel vier bis sieben knüpfen an diese Auflistung an und öffnen Perspektiven für die Zukunft der Masterstudiengänge in der Fremdsprachendidaktik. Sie betreffen das Herzstück der vorher verzeichneten Ausbildungsmodule, wobei es nun um eine qualitative Analyse geht. Diese Analyse basiert auf einer ,Triangulation' der Perspektiven der Dozenten, der Studenten und der Forscher. Ziel der Verknüpfung der drei Perspektiven ist es, die Schnittstellen zwischen Lehre, Forschung, Studium, Ästhetik und performativen Künsten herauszuarbeiten. Diese unterschiedlichen Beleuchtungen werden durch Interviews festgehalten und untersucht. Die Ergebnisse weisen auf zwei Perspektiven hin: Die erste ist eine „Innen-Perspektive“, bei der es sich hier um diejenige der Dozenten als den Spezialisten der Fremdsprachendidaktik handelt. Sie wird mit der Sichtweise der Studenten verglichen. Bei dieser doppelten Innen-Perspektive geht es um die praktische Anwendungsfähigkeit und deren konkrete Auswirkung auf das pädagogische Geschehen. Die zweite Perspektive ist gleichsam eine „Außen-Perspektive“. Hier geht es um die Experten der Lehre und Forschung im theater-/dramapädagogischen oder im hochschuldidaktischen Bereich. Sie bringen ihre Standpunkte ein, und zwar aus der Sicht der Theaterwissenschaften, der Performance Studies und der Erziehungswissenschaften. Nach Fleiner sind die Studien im Bereich der Performance - insbesondere die von FischerLichte, aber auch von Sting z.B. - und der Ästhetik sehr komplementär zu den fachspezifischen Veröffentlichungen im Bereich Fremdsprachenpädagogik. Sie garantieren die Formulierung von "vertiefenden Empfehlungen und kritischkonstruktiven Impulsen" (211), die dazu verhelfen könnten, das Angebot in der Hochschulausbildung zu verbessern und die Forschungsmethoden in der Femdsprachendidaktik um weitere Modelle aus den Performance-Künsten zu erweitern.

Bei seiner Ergebniszusammenfassung benennt Fleiner durchaus einige Schwierigkeiten, die bei der Anwendung von dramapädagogischen Arbeitsweisen auftreten können, etwa in Bezug auf den Zeitrahmen, der Lehrenden zur Verfügung steht oder auch verbunden sind mit der Tatsache, dass Lehrende keine Ausbildung durchlaufen haben und sich autodidaktisch in die Dramapädagogik hinein bewegen.

Trotz dieser verschiedenen Schwierigkeiten weisen die Ergebnisse in ihrer großen Mehrheit, sowohl aus der Perspektive der Dozenten als auch aus der der Studierenden, hauptsächlich positive Aspekte dramapädagogischer Ansätze auf (für beinahe $90 \%$ der Befragten überwiegen die Vorteile der dramapädagogischen Praxis).

Es lassen sich vor allem drei wichtige Lehren ziehen beim Lesen dieser Publikation, die Fleiner selbst in Form von desiderata für die pädagogische Ausund Fortbildung und für die Forschung zusammenfasst: 
1. Die Rolle der Performativität und der ästhetischen Erfahrung in der Unterrichtspraxis und beim Lernen, sowie für die Forschungsmethodologie (258; 264-269):

Fleiner stellt fest, dass die Aktionsforschung in Deutschland als „dirty science" betrachtet wird. Die ermutigenden Ergebnisse seiner Studie weisen im Gegensatz dazu darauf hin, dass es wünschenswert wäre, erfahrungsbasierte Methodologien im Bereich der Pädagogik zu erweitern und die bislang geltenden Einteilungskriterien der wissenschaftlichen Disziplinen, die auf einer heute überholten Geschichte beruhen, zu überschreiten. In dem Zusammenhang stellt Fleiner die derzeit stattfindende systematische Trennung der Fächer in Frage. Er plädiert anstatt dessen dafür, dass ein neues transversales Methodenrepertoire entwickelt und eingesetzt werden sollte, das der immanenten Komplexität der Lernprozesse und der Lerninstitutionen Rechnung tragen könnte (s.u.). Die Per-Formanz geht durch Formen durch, setzt sie um, verformt sie, löst sie auf, erfindet sie neu. In diesem kreativen Prozess entsteht Sprache und sollte dementsprechend gelehrt und gelernt werden. Daher wäre es nach Meinung des Autors besonders wichtig, neue empirische Forschungsmethodologien zu entwickeln, die sich zur weiteren Erforschung eines dramapädagogischen Fremdsprachenunterrichts besonders eignen.

2. Bedürfnis nach crosscurricularen Angeboten auf intra- und interuniversitären Ebene im Bereich von Bildung und Wissenschaft (270-294):

Es gehe dabei darum, Vernetzungsprozesse im Dienste der Reflexivität sowie der pädagogischen Handlung dank gemischter Fachrichtungen und Curricula zu entfalten. Demnach sollten entsprechende Rahmenbedingungen geschaffen werden, die dies ermöglichen, wie zum Beispiel durch eine Begrenzung der Studentenanzahl in den Gruppen, einen entsprechenden Raum mit Möglichkeiten, sich zu bewegen etc. Kann dieses Vorhaben gelingen, so wird dabei nicht zuletzt auch das kreative Potential von Dozenten und Lernern weiter gefördert.

Besonders überzeugend sind die Team-Teaching Module, die Fleiner analysiert. Sie bieten eine Lösung in inhaltlicher, methodischer, organisatorischer und zwischenmenschlicher Hinsicht zur Bewältigung einer transdisziplinären Materie und sollten dementsprechend ausgebaut werden. Fleiner betont darüber hinaus die Notwendigkeit, eine adäquate Ausbildung für Hochschullehrende und auch Lehrer an Schulen einzurichten.

Es ist in der Tat zu begrüßen, dass Dozierende adäquat fort- bzw. ausgebildet werden, um eine ausreichende Expertise im performativen Feld zu entwickeln. Es fragt sich jedoch, was mit Fleiners Vorschlag genau gemeint sein kann, ein „entsprechendes Anforderungsprofil“ (286) für diesen spezifischen Bildungsbereich zu entwickeln. Dies könnte nämlich letztlich gegen seine eigene Vorstellung der Prinzipien eines emergenten und kreativen Fremdsprachenunterrichts verstoßen. Ist es nicht bereits faktisch so, dass ein Überangebot von Kompetenzrastern die Forschung und Pädagogik in zu stark normierte Schemata einsperrt? Jegliche Art neuer Lösungen und phänomenaler Prozesse wäre doch von vornherein durch solche detaillierten Deskriptoren ausgeschlossen. 
Widersprächen sie nicht den Intentionen, die man mit performativen Ansätzen assoziiert?

Der Hinweis auf Kompetenzraster wirft die Frage der Evaluation auf, die Fleiner im dramapädagogischen Kontext erwähnt, indem er verschiedene Möglichkeiten auflistet: a.) Evaluation des individuellen Verhaltens: Disziplin, Eigeninitiative, ästhetische Kompetenz, Reflexivitätskompetenz, Sprachkompetenz etc.; b.) Interaktionskompetenz: Toleranz, Fähigkeit, zuzuhören etc. Fleiner erwähnt aber die Grenzen eines solchen Evaluationsmusters: Wie sollen ästhetische Kompetenz, Kreativität usw. evaluiert werden? Vielleicht könnten phänomenologische Selbstevaluationsansätze des lernenden Subjekts Fleiner eine Antwort bringen, insofern sie es ermöglichen, von der normativen zur qualitativen Evaluation überzugehen. Was die Forschungs- und Unterrichtsmethodologie betrifft, so könnte sicher die von Fleiner vorgeschlagene „Seminarperformance“ (275) Ausbildung, Forschung und Evaluation verbinden. Der Autor schlägt alternative Formen der Evaluation vor wie zum Beispiel „fantasie-, kunsterfahrungs -und lernbiographiekultivierende Diagnoseaspekte“ (281), Feedback etc.

Schließlich sieht Fleiner die transversalen Schnittstellen nicht nur innerhalb einer Hochschule, sondern auch im inter-universitären Kontext. Er schlägt vor, Netzwerke mit Künstlern, Verbänden, Theatergruppen sowie Plattformen zur Performativität im Fremdsprachenunterricht (wie zum Beispiel Scenario) zu erweitern.

3. Die Bedeutung und die Wirkung einer verkörperten (embodied) Methodologie im Sekundarbereich und an der Universität.

Aus der Sicht der Dozenten und der Studenten löst Drama Hemmungen auf, wenn in Lehr- und Lernsituationen das Wort in der fremden Sprache ergriffen werden soll. Der Sprechakt ist dann kein Schulritual mehr, sondern er entsteht in der gemeinsamen Handlung unter Lernenden, so wie jede Kommunikationssituation im „echten Leben“ auch stattfindet. Das Drama ist eine lebendige Kunst, die auf der Emergenz des Wortes und des Gedankens in der Interaktion basiert. Die Interaktionen werden nach und nach zu inszenierten Kreationen im Raum umgewandelt. Die unterrichteten und erlernten Sprachen, um die es hier geht, sind ebenfalls lebende Sprachen. Im Schmelztiegel des „Lebendigen“ findet also die Verbindung zwischen Dramapädagogik und Fremdsprachenunterricht statt.

Fleiners Befragungen weisen auf transformatorische Prozesse hin, die typisch für das Lernen sind. Sie betreffen die Person, die Gemeinschaft (Zusammenhalt), das Fach (Kultur, Lexik, Syntax, Phonetik/Phonologie) und auch die Methodologie (Ausbildung und Übertragung aufdie Unterrichtspraxis). Die Interaktionen in den Gesprächen werden sinngebend, dank des individuellen und kollektiven Imaginären der Akteure (194). Sie wecken Emotionen und unterstützen Memorierungsprozesse. Die Studierenden nehmen die Sprache in ihrer Unmittelbarkeit wahr und machen somit Lernerfahrungen, die sie auf traditionellem Wege nicht hätten machen können.

Das Ziel dieses Werkes, fachliche und formelle Grenzen zu überschreiten 
- indem der Autor Forschung und Ausbildung in der Fremdsprachendidaktik verbindet und dabei die Wirkung performativer Lehr- und Lernansätze untersucht - ist nicht nur für den deutschsprachigen Raum, sondern auch für Frankreich und sicherlich auch für viele weitere Länder von großer Relevanz. Fleiners Einladung, die performativen Wissenschaften im Hochschulbereich systematischer zu implementieren, sollte die methodologischen, universitären und geographischen Grenzen überschreiten. Fleiners innovative Empfehlungen sollten auf internationaler Ebene dringend umgesetzt werden, etwa auch in Verbindung mit mit den komplementären Forschungen von Irwin (2004) (A/R/Tography) und Varela (1989) (Enaktion). Fächerübergreifende Theoriebildungen, wie Aden (2008; 2015; in press) es vorschlägt, könnten die Fremdsprachendidaktik immens bereichern.

\section{Bibliographie}

Aden, Joëlle (in press): Developing empathy through theatre: a transcultural perspective in Second Language Education. In: Schewe, Manfred \& Crutchfield, John (Hrsg.): Going Performative in Intercultural Education. International contexts - theoretical perspectives - models of practice. Bristol: Multilingual Matters

Aden, Joëlle (2015): Théâtre et didactique des langues. In: Blanchet, Philippe \& Chardenet, Patrick (Hrsg.): Guide pour la recherche en didactique des langues et des cultures. Approches contextualisées. Paris: Édition des archives contemporaines, 422-437

Aden, Joëlle (2008): Apprentissage des langues et pratiques artistiques. Paris: Le Manuscrit

Austin, John L. (1962/1979): Quand dire, c'est faire. Paris: Folio Essais

Austin, John L. (1962/1972): Zur Theorie der Sprechakte. Stuttgart: Reclam

Böhme, Gernot (2013): Atmosphäre. Essays zur neuen Ästhetik. Berlin: Suhrkamp

Dewey, John (1934/1980): Art as experience: New York: Berkley Publishing Group

Defeu, Bernard (1996): Les approches non conventionnelles des langues étrangères. Paris: Hachette

Even, Susanne (2003): Drama Grammatik. Dramapädagogische Ansätze für den Grammatikunterricht Deutsch als Fremdsprache. München: iucidium

Feldhendler, Daniel (2009): Das Leben in Szene setzen. Wege zu einer relationellen Sprachdramaturgie. In: Scenario III/1, 50-69

Fischer-Lichte, Erika (2012): Performativität. Eine Einführung. Bielefeld: Transkript

Fischer-Lichte, Erika (2005): Performativität/performativ. In: Fischer-Lichte, Erika; Kolesch, Doris \& Warstat, Matthias: Metzler Lexikon Theatertheorie. Stuttgart et al.: Metzler, 234-242 
Hentschel, Ulrike (2010): Theaterspielen als ästhetische Bildung. Berlin: Schibri

Huber, Ruth (2003): Im Haus der Sprache wohnen. Wahrnehmung und Theater im Fremdsprachenunterricht. Tübingen: Max Niemeyer

Irwin, Rita (2004): A/R/Tography. A Metonymic métissage. In: Irwin, Rita \&

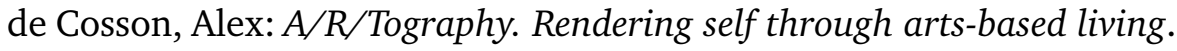
Vancouver, British Columbia, Canada: Educational Press, 27-38

Kessler, Benedikt (2008): Interkulturelle Dramapädagogik. Dramatische Arbeit als Vehikel des interkulturellen Lernens im Fremdsprachenunterricht. Frankfurt/M. et al.: Peter Lang

Koch, Gerd (2011): Zur Theaterpädagogik in der Bundesrepublik Deutschland. Ein Überblick. In: Scenario V/1, 76-94

Küppers, Almut; Schmidt, Torben und Walter, Maik (Hrsg.) (2011): Inszenierungen im Fremdsprachenunterricht. Kempten: Diesterweg

Lutzker, Peter (2007): The Art of Foreign Language Teaching. Improvisation and Drama in Teacher Development and Language Learning. Tübingen et al.: Francke

Sambanis, Michaela (2013): Fremdsprachenunterricht und Neurowissenschaften. Tübingen: Narr

Schewe, Manfred (2011): Die Welt auch im fremdsprachlichen Unterricht immer wieder neu verzaubern. In: Küppers, Almut; Schmidt, Torben \& Walter, Maik (Hrsg.): Inszenierungen im Fremdsprachenunterricht. Kempten: Diesterweg, 20-31

Schewe, Manfred (2010): Dramapädagogik. In: Surkamp, Carola (Hrsg): Metzler Lexikon Fremdsprachendidaktik. Ansätze, Methoden, Grundbegriffe. Stuttgart et al.: Metzler 38-41

Schewe, Manfred (1993): Fremdsprache inszenieren. Zur Fundierung einer dramapädagogischen Lehr- und Lernpraxis. Oldenburg: Universität Oldenburg, Zentrum für Berufpraxis

Streisand, Marianne (2012): Geschichte der Theaterpädagogik im 20. und 21. Jahrhundert. In: Nix, Christoph; Sachser, Dietmar \& Streisand, Marianne: Theaterpädagogik (Lektionen). Berlin: Theater der Zeit, 14-35

Sting, Wolfgang (2012): Performative Praxen und Theaterpädagogik. In: Sting, Wolfgang et al.: TUSCH: Poetiken des Theatermachens, Werkbuch für Theater und Schule. Bobingen: Kessler Druck und Medien/Kopaed. 119-126

Sting, Wolfgang (2003): Ästhetische Kompetenz. In: Koch, Gerd \& Streisand, Marianne: Wörterbuch der Theaterpädagogik. Milow et al.: Schibri, 11-12

Varela, Francisco (1989): Invitation aux sciences cognitives, Paris: Edition Seuil, Collection Points Sciences

Welsch, Wolfgang (2012): Blickwechsel. Neue Wege der Ästhetik. Stuttgart: Reclam

Welsch, Wolfgang (2010): Ästhetisches Denken. Stuttgart: Reclam

Welsch, Wolfgang (1996): Grenzgänge der Ästhetik. Stuttgart, Reclam 
Wulf, Christoph \& Zirfas, Jörg (2007): Performative Pädagogik und performative Bildungstheorien. Ein neuer Fokus erziehungswissenschaftlichern Forschung. In: Wulf, Christoph \& Zirfas, Jörg: Pädagogik des Performativen. Theorien, Methoden, Perspektiven. Weinheim et al.: Belz, 7-40 